\title{
0 papel da atenção na fisioterapia neurofuncional
}

\author{
The role of attention in neurofunctional physical therapy
}

Andréa Lúcia Sério Bertoldi ${ }^{1}$, Vera Lúcia Israel² Iverson Ladewig $^{3}$

Estudo desenvolvido no

Laboratório CECOM - Centro

de Estudos do Comportamento Motor da Universidade Federal do Paraná - Curitiba, PR, Brasil.

${ }^{1}$ Fisioterapeuta; Mestre; Pesquisadora do Laboratório CECOM - Centro de Estudos do Comportamento Motor da Universidade Federal do Paraná (UFPR); Doutoranda do Departamento de Educação Física da UFPR - Curitiba (PR), Brasil.

${ }^{2}$ Fisioterapeuta; Professora Doutora do Departamento de Fisioterapia do Curso de Fisioterapia da UFPR, Setor Litoral - Curitiba (PR), Brasil.

${ }^{3}$ Educador Físico; Professor Doutor do Departamento de Educação Física, Curso de Educação Física; Pesquisador do laboratório CECOM da UFPR Curitiba (PR), Brasil.

\section{ENDEREÇO PARA \\ CORRESPONDÊNCIA:}

Andréa Lúcia Sério Bertoldi Rua Johann Sebastian Bach, 45, Vista Alegre - CEP: 80820-140 - Curitiba (PR), Brasil - E-mail: seriobertoldi@gmail.com

$$
\begin{aligned}
& \text { APRESENTAÇ̃OO } \\
& \text { jan. } 2010 \\
& \text { ACEITO PARA PUBLICAÇÃO } \\
& \text { ago. } 2010
\end{aligned}
$$

\section{FONTE DE FINANCIAMENTO: nenhuma}

\section{CONFLITO DE INTERESSE:} nada a declarar
RESUM0: Este estudo apresenta uma revisão teórica sobre o papel da atenção na realização de tarefas motoras, adotando-se a perspectiva do paradigma sistêmico aplicado ao comportamento motor e sua relação com a abordagem do controle motor na fisioterapia neurofuncional. Foram consultados os bancos de dados Medline, PubMed, LILACS, SciELO, e PEDro, incluindo artigos de 1990 a 2009. Nos estudos pesquisados, observou-se o entendimento da atenção como um agente mediador entre o indivíduo e o ambiente no processo de auto-organização dos subsistemas orgânicos durante a realização de uma tarefa motora. Foram identificadas similaridades entre esta perspectiva teórica sobre a atenção e os pressupostos que fundamentam a abordagem do controle motor na fisioterapia neurofuncional, evidenciando caminhos de investigação científica no sentido de ser mais bem compreendido o papel da atenção na adaptação do sistema neuromotor de pessoas com lesão neurológica e sua efetividade na promoção do movimento funcional humano.

Descritores: atenção; fisioterapia; neurologia; teoria de sistemas; comportamento.

ABSTRACT: This study presents a theoretical review of attention in the creation of strategies of movement control from the perspective of the systemic paradigm applied to motor behavior and its relationship with the motor control approach in neurofunctional physical therapy. The following databases were consulted Medline, PubMed, LILACS, SciELO, and PEDro, including articles from 1990 to 2009. Research has shown the understanding of attention as a mediator agent between a subject and the environment in the process of self-organization of organic sub-systems during the realization of a motor task. Similarities between this theoretical perspective of attention and the assumptions that support the motor control in the neurofunctional physical therapy approach have been identified, evidencing paths of scientific investigation for a better comprehension about the attention role on neuromotor system adaptation of individuals with neurological lesions and its efficiency to promote human functional movement.

KeYwords: attention; physical therapy; neurology; systems theory, behavior. 


\section{INTRODUCÃO}

Procedimentos terapêuticos neurológicos se fundamentam em abordagens teóricas sobre como o sistema nervoso central (SNC) controla os movimentos ${ }^{1}$. O desafio da transferência funcional, ainda não-solucionado nas abordagens terapêuticas fundamentadas na concepção de organização hierárquica do SNC, tem impulsionado a tendência de estudos do controle motor na fisioterapia neurofuncional ${ }^{1,2}$. Nesta abordagem, presume-se que, além de entender-se como o SNC controla os movimentos, deve-se compreender os problemas enfrentados por este nesse controle ${ }^{2,3}$.

Entre os problemas enfrentados pelo SNC para controlar movimentos, destaca-se o processo de formulação de estratégias para a realização de movimentos funcionais, isto é, que atinjam o objetivo proposto com o menor dispêndio de energia possível ${ }^{3}$. A seletividade de atenção é considerada um fator importante neste processo, na medida em que é entendida como um agente mediador entre a captação das informações ambientais e a realização do movimento ${ }^{4,5}$.

Os pressupostos teóricos, que definem a atenção como um elo entre organismo e ambiente, estão fundamentados no paradigma sistêmico ${ }^{6}$ aplicado à área do comportamento motor ${ }^{7}$. Essa visão sobre o papel da atenção para o movimento humano permite relações com a abordagem do controle motor na fisioterapia neurofuncional ${ }^{1,2}$. Entretanto, a atenção ainda é pouco investigada na Fisioterapia e identifica-se a necessidade de atualização teórica sobre esta temática. Diante disso, este estudo tem como objetivo apresentar uma revisão teórica sobre a atenção, sob o ponto de vista do paradigma sistêmico aplicado à área do comportamento motor, e sua relação com pressupostos teóricos da abordagem do controle motor na fisioterapia neurofuncional.

\section{MATERIAL E MÉTODOS}

Foi conduzida uma busca em banco de dados computadorizado para identificar artigos científicos relevantes ao estudo, incluindo Medline, PubMed, LILACS, SciELO e PEDro. Os artigos foram selecionados entre 1990 e 2009, atendendo-se aos seguintes critérios de inclusão: adotar uma abordagem teórica sistêmica em relação aos efeitos da atenção e/ou percepção no comportamento motor humano; adotar uma abordagem teórica sistêmica em relação ao(s) procedimento(s) terapêutico(s) utilizado(s) na fisioterapia neurológica e descrever os efeitos do direcionamento da atenção no comportamento motor de pessoas com lesão neurológica. Nos 52 artigos selecionados, realizou-se uma busca manual de referências apresentadas, considerando os mesmos critérios de inclusão. As palavras-chave utilizadas foram: atenção, percepção, comportamento motor, controle motor, paradigma sistêmico, sistemas dinâmicos e fisioterapia neurológica nos idiomas português e inglês.

\section{RESULTADOS E DISCUSSÃO}

Abordagem sistêmica do papel da atenção na área do comportamento motor

O comportamento motor é uma área de conhecimento que envolve os estudos do desenvolvimento motor, aprendizagem motora e controle motor ${ }^{7}$. Verificase, nesta área, a fundamentação teórica sobre o papel da atenção humana para o controle dos movimentos, apoiada em uma abordagem sistêmica ${ }^{4,5}$, na qual todo ser vivo está sujeito à troca de informação e matéria constantemente com o meio ${ }^{7-9}$. Neste contexto, a seletividade da atenção bem como os mecanismos perceptivos advindos dos sistemas visual, vestibular e somatossensorial assumem papel importante no trabalho cooperativo de comandos centrais e periféricos para controle motor do organismo em contato com o meio externo ${ }^{5,10,11}$.

Esta persperctiva teórica assume que o controle dos movimentos ocorre por meio da organicidade dos sistemas corporais, atuando em conjunto para selecionar as opções percebidas pelo organismo como as mais adequadas para uma ação, entre um infinito número de graus de liberdade possíveis ${ }^{9,12}$. Este grande número de possibilidades de combinações dos movimentos é reduzido a um conjunto maleável de agrupamentos musculares, os quais podem executar movimentos coordenados, mesmo sem comandos neurais detalhados vindos do $\mathrm{SNC}^{11,12}$. Este conjuto de músculos, articulações e segmentos corporais são controlados como um todo pelo sistema na realização de uma tarefa, e são chamados de estruturas coordenativas que atuam como sinergias funcionais, definindo estratégias neurais de acoplamento dos movimentos em articulações multissegmentares. O controle motor emerge, então, de um processo de auto-organização por meio da adaptação do sistema às condições ambientais e às exigências da tarefa que o executante se propõe a fazer ${ }^{10-12}$.

O pressuposto de que a emergência dos padrões motores é fruto de autoorganização do sistema fundamenta o entendimento da atenção humana no contexto do ciclo percepção-ação ${ }^{4,5,13,14}$. Nesta perspectiva, a atenção não está mais restrita a elementos internos ou externos ao organismo, mas é exatamente na relação entre eles que desempenha o seu papel auto-organizador ${ }^{5,13}$. Em sua interação com o meio, o ser humano busca um conjunto de informações que lhe permita atingir objetivos funcionais em sincronia com o ambiente circundante. Esta interação envolve a intenção do executante, a captação da informação do ambiente e a organização dos comandos para resposta $^{5}$. A atenção é considerada como um agente mediador deste processo ${ }^{15,16}$. É justamente a organização do ambiente, captada pelos sistemas perceptivos dos ser humano, e seus estados disposicionais para a ação que determinam a qualidade e a pertinência da estratégia de movimento utilizada e, por consequência, o conjunto de adaptações necessarias à funcionalidade da ação ${ }^{11,14,17,18}$.

Embora o organismo, agindo como um sistema integrado, apresente uma autoorganização que naturalmente emerge no momento em que interage com o meio, nem sempre esta organização ocorre de maneira eficiente, sob o ponto de vista da funcionalidade motora ${ }^{18}$. A dificuldade da formulação de estratégias funcionais para uma ação está realacionada à inabilidade perceptiva do sistema ${ }^{5}$, isto é, à dificuldade de reconhecimento das possibilidades de ação disponíveis para serem utilizadas em um dado contexto, em função das restrições impostas pelo organismo, pela própria tarefa, pelo ambiente ou pela interação dos mesmos ${ }^{17-19}$. 
A perspectiva teórica da atenção como um elo entre a percepção e a ação é apresentada como uma posssibilidade de flexibilizar a organização existente, para que novas formas de comportamento e soluções de problemas motores possam emergir $^{5,13,15}$.

Outra questão enfatizada é o fato deste processo, de captar informações do meio para a realização de ações funcionais, não ser necessariamente consciente, resultado de processos cognitivos complexos, mas fruto do ciclo percepçãoação que se estabelece com diferentes níveis de envolvimento cognitivo do ser humano ${ }^{10,11}$. Desse modo, a atenção não é mais entendida sob o ponto de vista estrito de processos controlados ou automáticos, o primeiro enfatizando o controle central e o segundo o controle periférico do movimento humano, mas passa a ser compreendida na interdependência destes processos, assumindo seu papel organizador nos mecanismos perceptivos do indivíduo ${ }^{14,20}$.

Abordagem sistêmica do movimento aplicada à área da fisioterapia neurofuncional e perspectivas de relação com o papel da atenção no comportamento motor humano

$\mathrm{Na}$ abordagem sistêmica ou abordagem do controle motor na fisioterapia neurofuncional ${ }^{1,2}$, destaca-se a prerrogativa teórica de que a execução de atividades motoras em ambientes abertos deve ser comandada por um processo baseado mais em mecanismos de flexibilização e adaptação das ações motoras do que em reações pré-determinadas de estímulo e resposta $^{1,2,21}$. Considera-se o fato de que muitos movimentos não são totalmente dependentes de padrões pré-planejados de resposta e admite-se a possibilidade do desenvolvimento de adaptação de respostas do sistema neural, ou seja, é reconhecida a característica de plasticidade deste sistema ${ }^{22-24}$. Nesta abordagem, enfatiza-se a visão ativa da aprendizagem motora, decorrente do entendimento de que o movimento humano intencional nada mais é que o resultado de estratégias criadas pelos subsistemas do organismo, os quais surgem da interação de nossas necessidades com o ambiente. Portanto, um movimento é considerado funcional não por sua correspondência a um modelo ideal, mas pelo fato de estar bem ajustado à solução de um determinado problema ou desafio motor ${ }^{25,26}$.

Outro pressuposto apresentado é o fato de a lesão neurológica ser considerada como uma restrição imposta ao organismo, assim, a emergência dos movimentos preferenciais de um sistema é considerada uma forma de auto-organização percebida por este sistema ${ }^{1,2,27}$. Esta ideia implica no reconhecimento de que movimentos pouco funcionais, comumente observados após uma lesão neurológica, não são apenas o resultado da perda de algum tecido neural, como em uma relação estrita de causa e efeito, mas, também, das tentativas dos tecidos remanescentes de compensarem essa perda ou desequilíbrio tecidual para promover o controle motor ${ }^{1,2,28,29}$.

Destaca-se ainda a importância da prática de intervenções promotoras de adaptabilidade dos movimentos a diferentes situações ambientais ${ }^{30,31}$. De acordo com esta perspectiva, o conceito de que pessoas com lesão neurológica aprendem movimentos pela repetição dos padrões de estimulação que desencadeiam movimentos normais, fundamentados na psicologia behaviorista tradicional ${ }^{1,2}$, apresenta limitações para promover a flexibilidade necessária à adaptação do movimento humano, sendo identificada a necessidade do desenvolvimento das estratégias de intervenção que considerem a variabilidade do movimento para resolver desafios motores em ambientes abertos, isto é, sujeitos a variações ${ }^{31-33}$. Admite-se que embora a especificidade da prática seja um pré-requisito importante para a aprendizagem de uma tarefa, ela deva considerar o desenvolvimento de diferentes estratégias de controle dos movimentos ${ }^{1,2,33}$.

Percebe-se, nestas prerrogativas teóricas apresentadas sobre a abordagem sistêmica ou do controle motor na fisioterapia neurofuncional, uma relação estreita com a fundamentação teórica apresentada anteriormente sobre o papel da atenção no comportamento motor humano, sobretudo em seu potencial organizador do ciclo percepção-ação, isto é, na perspectiva da seletividade de atenção influenciar a detecção e utilização coerente das informações sensoriais disponíveis no ambiente para a realização de uma ação, facilitando a emergência de soluções motoras funcionais. Esta relação abre um amplo caminho de investigação sobre o papel da atenção no comportamento motor de pessoas com lesão neurológica. Entretanto, poucos estudos têm investigado os efeitos da seletividade de atenção no comportamento motor desta população.

Entre os 52 artigos revisados neste estudo, apenas cinco relatavam estudos clínicos sobre os efeitos da atenção em comportamentos motores de pessoas com lesão neurológica. A Tabela 1 apresenta a síntese dos objetivos, métodos e principais resultados destes artigos. Verifica-se que três estudos compararam o efeito dos focos de atenção interno e externo em tarefas funcionais com adultos com doença de Parkinson ${ }^{34,35}$ e com acidente vascular encefálico $^{36}$, tendo sido verificados nos três estudos benefícios do foco externo de atenção para o controle motor. Um estudo demonstrou ganhos funcionais em comportamentos motores de um adulto com lesão medular submetido a um programa de intervenção, que direcionou a atenção para parâmetros de amplitude, velocidade e tensão dos movimentos ${ }^{37}$, e foram demonstradas diferenças estatisticamente significativas em favor do uso de estratégicas cognitivas de direcionamento de atenção (dicas de aprendizagem) em um estudo que investigou as alterações do esquema e da imagem corporal de crianças com paralisia cerebral $^{38}$.

Embora em diversos estudos ${ }^{39-41}$ seja demonstrada a relação entre seletividade de atenção e a adaptabilidade motora, é preciso avançar na investigação científica sobre o papel da atenção no estabelecimento das estratégias de movimento, sobretudo com pessoas que apresentam lesão neurológica. Há necessidade de maiores esclarecimentos sobre a maneira como o organismo se adapta às alterações nos parâmetros dos estímulos sensoriais ${ }^{42-45}$, bem como sobre as estratégias de direcionamento de atenção a serem manipulados em procedimentos fisioterapêuticos ${ }^{37,38}$. Estas, entre outras questões, demonstram perspectivas de estudos futuros, no sentido de melhor compreender o papel da seletividade da atenção na adaptação do sistema neuromotor de pessoas com lesão neurológica e sua efetividade na promoção do movimento funcional humano. 
Tabela 1. Síntese de experimentos sobre os efeitos da atenção em comportamentos motores de pessoas com lesão neurológica

\begin{tabular}{|c|c|c|c|}
\hline Autores & Objetivo e amostra & Procedimentos e instrumentos & Principais resultados \\
\hline $\begin{array}{l}\text { Wulf; } \\
\text { Landers; } \\
\text { Lewthwait e } \\
\text { Tollner }^{34}\end{array}$ & $\begin{array}{l}\text { Avaliar os efeitos do foco } \\
\text { de atenção no equilíbrio } \\
\text { de } 14 \text { idosos com doença } \\
\text { de Parkinson idiopática. }\end{array}$ & $\begin{array}{l}\text { Tarefa experimental de manutenção do } \\
\text { equilíbrio em uma superfície instável em três } \\
\text { condições: com foco de atenção interno ( para } \\
\text { os pés); com foco externo (para a superfície) } \\
\text { e controle (sem foco de atenção específico). } \\
\text { Variáveis do equilíbrio foram medidas por meio } \\
\text { de plataforma de força. }\end{array}$ & $\begin{array}{l}\text { O uso de foco externo } \\
\text { diminuiu a oscilação corporal } \\
\text { quando comparado com foco } \\
\text { interno ou controle. Não } \\
\text { houve diferença entre foco } \\
\text { interno e controle. }\end{array}$ \\
\hline $\begin{array}{l}\text { Bertoldi, } \\
\text { Ladewig e } \\
\text { Israel }^{37}\end{array}$ & $\begin{array}{l}\text { Avaliar os efeitos da } \\
\text { atenção para parâmetros } \\
\text { do movimento em } \\
\text { comportamentos } \\
\text { motores funcionais de } \\
\text { um adulto com lesão }\end{array}$ & $\begin{array}{l}\text { Tratamento com } 32 \text { sessões compostas de } \\
\text { movimentos randômicos de estabilização, } \\
\text { manipulação e locomoção com atenção } \\
\text { direcionada para a parâmetros de amplitude, } \\
\text { velocidade e tensão. Foram realizados pré- } \\
\text { teste, pós-teste e retenção dos comportamentos }\end{array}$ & $\begin{array}{l}\text { Houve evolução no nível } \\
\text { de classificação funcional } \\
\text { entre o pré- e o pós-teste nos } \\
\text { comportamentos C1 e C2. Os } \\
\text { resultados foram mantidos no } \\
\text { teste de retenção. }\end{array}$ \\
\hline
\end{tabular}
medular traumática funcionais C1 (transferência da posição deitada adquirida (tetraparesia), para a sentada) e C2 (da posição sentada para a com alta de tratamento ortostática com auxílio), com uso de escala de fisioterapêutico. avaliação funcional ${ }^{37}$ ).

Bertoldi, Verificar a influência Tratamento com quatro sessões composto por Ladewig e do uso de estratégias Israel ${ }^{38} \quad$ cognitivas de atenção (dicas de aprendizagem) em variáveis da tarefas lúdicas de estabilização, locomoção e manipulação, utilizando dicas de aprendizagem para partes do corpo (Grupo CD) e as mesmas atividades sem direcionamento percepção corporal de 22 de atenção (Grupo SD). Variáveis da percepção crianças com deficiência corporal foram avaliadas em pré-teste, pós-teste motora, selecionadas por similaridade do quadro funcional e dividas aleatoriamente em dois grupos: $C D$ (com direcionamento de atenção) e SD (sem direcionamento de atenção).

Landers; Comparar os efeitos de Wulf; $\quad$ instruções verbais com Wallmann e focos externo, interno Guadagnoli ${ }^{35}$ e sem foco de atenção específico em variáveis do equilíbrio de 22 adultos com doença de Parkinson idiopática. e teste de retenção com aplicação do teste de identificação das partes do corpo, teste de percepção cinética e teste de percepção crítica das partes do corpo ${ }^{38}$.

Foram verificadas diferenças significativas com superioridade de aproveitamento do Grupo CD, nas variáveis de percepção cinética e de percepção crítica das partes do corpo no pósteste. Os resultados foram mantidos no teste de retenção.

Tarefa experimental de manutenção do equilíbrio em uma superfície instável em três condições: com instrução de foco de atenção interno (para os pés); com instrução de foco externo (para a superfície) e sem instrução de foco de atenção específico (controle). Posturografia dinâmica computadorizada foi utilizada para avaliar variáveis do equilíbrio.

Tarefa experimental de alcance com o membro superior afetado foi avaliada sob duas condições: com foco interno (atenção direcionada para o braço que realizava a ação) e com foco externo (atenção direcionada para a tarefa). Cinemática tridimensional foi utilizada para avaliar variáveis temporais e espaciais do movimento.
Houve aumento de oscilação corporal com instrução de foco interno. Quando considerados apenas os sujeitos com história de quedas, verificou-se que as instruções de foco externo resultam em menor oscilação corporal quando comparadas com foco interno ou sem instrução de foco de atenção específico.

Foram verificadas diferenças significativamente menores no tempo de movimento e maior pico de velocidade quando a tarefa funcional de alcançar foi realizada com foco externo. movimento de 16 adultos com acidente vascular encefálico e 17 adultos sem comprometimento neurológico (controle). 


\section{CONSIDERACÕ̃ES FINAIS}

Este estudo apresentou uma revisão teórica sobre a perspectiva sistêmica do papel da atenção no comportamento motor e sua relação com a abordagem do controle motor na fisioterapia neurofuncional. Observou-se a ênfase no atual entendimento da atenção como elo entre os recursos inerentes ao funcionamento de organismos vivos e as variações do ambiente para a realização de uma tarefa, no chamado ciclo percepção-ação. Esta perspectiva teórica sobre a atenção evidencia a sua relação com a abordagem do controle motor na Fisioterapia, na medida em que esta abordagem está fundamentada na compreensão do movimento funcional humano, como resultado da adaptabilidade do organismo, agindo como um sistema na elaboração de estratégias para solucionar desafios motores. Esta relação apresenta caminhos de investigação científica a serem desenvolvidos, os quais poderão contribuir para a fundamentação de novos procedimentos de intervenção condizentes com a atual tendência interdisciplinar da Neurofisiologia e do Comportamento Motor aplicados à área da Fisioterapia Neurofuncional.

\section{REFERENNCIAS}

1. Carr JH, Shepherd RB. The changing face of neurological rehabilitation. Rev Bras Fisioter. 2006;10(2):147-56.

2. Gordon J. Assumptions underlying physical therapy interventions: theoretical and historical perspectives. In: Carr JH, Shepherd RB, editors. Movement science foundations for physical therapy in Rehabilitation. $2^{\text {nd }}$ ed. Gaithersburg: Aspen; 2000. p.1-31.

3. Kelso LA, Zanonne PG. Coordination dynamics of learning and transfer across different effector systems. J Exp Psychol Hum Percept Perform. 2002;28(4):776-97.

4. McNevin NH, Wulf G, Carlson C. Effects of attentional focus, self-control, and dyad training on motor learning: implications for physical therapy. Phys Ther. 2000;80(4):373-85.

5. Pellegrini AM. Revisitando a atenção. In: Teixeira LA, editor. Avanços em comportamento motor. São Paulo: Movimento; 2001. p.147-65.

6. Schoner G. Recent development and problems in human movement science and their conceptual implications. Ecol Psycol. 1995;7(4):291-314.

7. Tani G, Freudenheim AM, Meira Júnior CM, Corrêa UC. Aprendizagem Motora: tendências, perspectivas e problemas de investigação. Rev Paul Educ Fis. 2004;18(N esp):55-72.

8. Davis W, Burton AW. Ecological task analysis: translating movement behavior theory into practice. Adapt Phys Act Q. 1991;8(2):154-77.

9. Newell KM, Broderick MP, Deutsch KM, Slifkin AB. Task goals and change in dynamic degrees of freedom with motor learning. J Exp Psychol Hum Percept Perform. 2003;29(2):379-87.

10. Paine RW, Tani G. How hierarchical control selforganizes in artificial adaptative systems. Adap Behav. 2005;13(3):211-25

11. Decety J, Grezes, J. Neural mechanisms subserving the perception of human actions. Trends Cogn Sci. 1999;3(5):172-8.
12. Berthouze L, Lungarella M. Motor skill acquisition under environmental perturbations: on the necessity of alternate freezing and freeing degrees of freedom. Adap Behav. 2004;12(1):47-64.

13. Michaels CF, Withagen R, Jacobs DM, Zaal FTJM, Boners RM. Information, perception and action: a reply to commentators. Ecol Psycol. 2001;13(3):227-44.

14. Johnston LM, Burns YR, Brauer SG, Richards CA. Differences in postural control and movement performance during goal directed reaching in children with developmental coordination disorder. Hum Movement Sci. 2002;21(5):583-601.

15. Court MJL, Bennett SJ, Williams AM, Davids K. Effects of attentional strategies and anxiety constraints on perceptual-motor organization of rhythmical arm movements. Neurosci Lett. 2005;384(1):17-22.

16. Ladewig I. A importância da atenção na aprendizagem de habilidades motoras. Rev Paul Educ Fis. 2000;14 Suppl 3:62-71.

17. Baran Y, Miller A. Auditory feedback control for improvement of gait in patients with multiple sclerosis. J Neurol Sci. 2007;254(1):90-4.

18. Wu C, Trombly CA, Lin K. A kinematic study of contextual effects on reaching performance in persons with and without stroke: influences of object availability. Arch Phys Med Rehab. 2000;81(1):95-101.

19. Brianeze ACGS, Cunha A, Peviani SM, Miranda VCR, Tognetti VBL, Rocha NACF, et al. Efeito de um programa de fisioterapia funcional em crianças com paralisia cerebral associado a orientações aos cuidadores: estudo preliminar. Fisioter Pesq. 2009;16(1):40-5.

20. Volpe BT, Krebs HI, Hogan N. A novel approach to stroke rehabilitation: robot-aided sensorimotor stimulation. Neurology. 2000;54:1938-44.

21. Ashford D, Bennet S, Davids K. Observational modeling effects for movement dynamics and movement outcome measures across differing task constraints: a metaanalysis. J Motor Behav. 2006;38:185-205. 


\section{Referências (cont.)}

22. Hoare B, Imms C, Carey L, Wasiak J. Constraint-induced movement therapy in the treatment of the upper limb in children with hemiplegic cerebral palsy. Clin Rehabil. 2007;21(8):675-85

23. Liepert J, Uhde I, Graf S. Motor cortex plasticity during forced-use therapy in stroke patients: a preliminary study. J Neurol. 2001;248(4):315-21.

24. Gauthier LV, Taub E, Perkins C, Ortmann M, Mark VW, Uswatte G. Remodeling the brain: plastic structural brain changes produced by different motor therapies after stroke. Stroke. 2008;39(5):1520-5.

25. Salbach NM, Mayo NE, Wood-Dauphinee S. A taskoriented intervention enhances walking distance and speed in the first year post stroke: a randomized controlled trial. Clin Rehabil. 2004;18(5):509-18.

26. Higgins J, Salbach NM, Wood-Dauphinee S, Richards CL, Cote R, Mayo NE. The effect of a taskoriented intervention on arm function in people with stroke: a randomized controlled trial. Clin Rehabil. 2006;20(4):296-310.

27. Scholz P, Schöner G, Hsu WL, Jeka JJ, Horak F, Martin $\mathrm{V}$. Motor equivalent control of the center of mass in response to support surface perturbations. Exp Brain Res. 2007;180(1):163-79.

28. Celnik P, Hummel F, Harris-Love M, Wolk R, Cohen LG. Somatosensory stimulation enhances the effects of training functional hand tasks in patients with chronic stroke. Arch Phys Med Rehab. 2007;88(11):1369-76.

29. Dean CM, Richards CL, Malouin F. Task-related training improves performance of locomotor tasks in chronic stroke. A randomized controlled pilot study. Arch Phys Med Rehab. 2000;81(4):409-17.

30. Beekhuizen KS, Field-Fote EC. Massed practice versus massed practice with stimulation: effects on upper extremity function and cortical plasticity in individuals with incomplete cervical spinal cord injury. Neurorehabil Neural Repair. 2005;19(1):33-45.

31. Lin KC, Wu CY, Wei TH, Lee CY, Liu JS. Effects of modified constraint-induced movement therapy on reach-to-grasp movements and functional performance after chronic stroke: a randomized controlled study. Clin Rehabil. 2007;21(12):1075-86.

32. Hakkennes S, Keating JL. Constraint-induced movement therapy following stroke: a systematic review of randomised controlled trials. Aust J Phys. 2005;51(4):221-31.

33. Salbach NM, Mayo NE, Wood-Dauphinee S. A taskoriented intervention enhances walking distance and speed in the first year post stroke: a randomized controlled trial. Clin Rehabil. 2004;18(5):509-18.
34. Wulf G, Landers M, Lewthwaite R, Tollner T. External focus instructions reduce postural instability in individuals with Parkinson disease. Phys Ther. 2009;89(2):162-8.

35. Landers M, Wulf G, Wallmann H, Guadagnoli M. An external focus of attention attenuates balance impairment in patients with Parkinson's disease who have a fall history.Physioterapy. 2005;91(3):152-8.

36. Fasoli SE, Trombly CA, Ticle-Degned L, Nerfaellie MH. Effect of instructions on functional reach in persons with and without cerebrovascular accident. Am J Occup Ther. 2002;56(4):380-90.

37. Bertoldi ALS, Ladewig I, Israel VL. Efeitos da atenção dirigida para parâmetros da qualidade do movimento na recuperação neurofuncional. Fisiot Mov. 2008;21(3):19-25.

38. Bertoldi ALS, Ladewig, Israel VL. Influência da seletividade de atenção no desenvolvimento da percepção corporal de crianças com deficiência motora. Rev Bras Fisioter. 2007;11(4):319-24.

39. Wulf G, Shea C, Park JH. Attention and motor performance: preferences for and advantages of an external focus. Res Q Exercise Sport. 2001;72(4):335-44.

40. Zachry T, Wulf G, Mercer J, Bezodis N. Increased movement accuracy and reduced EMG activity as the result of adopting an external focus of attention. Brain Research Bulletin. 2005;67(2):304-9.

41. Janelle CM, Chapenoy JD, Coombes SA, Mousseau MB. Mechanics of attentional cueing during observational learning to facilitate motor skills acquisition. J Sports Sci. 2003;21(10):825-38.

42. Hur JJ. Review of research on therapeutic interventions for children with cerebral palsy. Acta Neurol Scand. 1995;91(6):423-32.

43. Tunik E, Ortigue S, Adamovich SV, Scott TG. Differential recruitment of anterior intraparietal sulcus and superior parietal lobule during visually guided grasping revealed by electrical neuroimaging. J Neurosci. 2008;28(50):16315-20.

44. Totsika V, Wulf G. The influence of external focus of attention in transfer to novel situations and skills. Res Quart Exercise Sport. 2003;74:220-5.

45. Gosselin-Kessiby N, Kalaska JF, Messier J. Evidence for a proprioception-based rapid on-line error correction mechanism for hand orientation during reaching movements in blind subjects. J Neurosci. 2009;29(11):3485-96. 